\title{
Older adults and high-risk medication administration in the emergency department
}

\author{
This article was published in the following Dove Press journal: \\ Drug, Healthcare and Patient Safety \\ 8 November 2017 \\ Number of times this article has been viewed
}

\author{
Mitchell Kim' \\ Steven H Mitchell' \\ Medley Gatewood' \\ Katherine A Bennett ${ }^{2}$ \\ Paul R Sutton ${ }^{3}$ \\ Carol A Crawford ${ }^{4}$ \\ Itay Bentov ${ }^{5}$ \\ Mamatha Damodarasamy ${ }^{2}$ \\ Stephen J Kaplan ${ }^{6}$ \\ May J Reed ${ }^{2}$ \\ 'Department of Emergency Medicine, \\ University of Washington, ${ }^{2}$ Division of \\ Gerontology and Geriatric Medicine, \\ Department of Medicine, University \\ of Washington, ${ }^{3}$ Division of General \\ Internal Medicine, Department of \\ Medicine, University of Washington, \\ ${ }^{4}$ School of Pharmacy, University \\ of Washington, ${ }^{5}$ Department of \\ Anesthesiology and Pain Medicine, \\ University of Washington, ${ }^{6}$ Section \\ of General, Thoracic and Vascular \\ Surgery, Department of Surgery, \\ Virginia Mason Medical Center, \\ Seattle, WA, USA
}

\begin{abstract}
Background: Older adults are susceptible to adverse effects from opioids, nonsteroidal antiinflammatory drugs (NSAIDs), and benzodiazepines (BZDs). We investigated factors associated with the administration of elevated doses of these medications of interest to older adults ( $\geq 65$ years old) in the emergency department (ED).

Patients and methods: ED records were queried for the administration of medications of interest to older adults at two academic medical center EDs over a 6-month period. Frequency of recommended versus elevated ("High doses" were defined as doses that ranged between 1.5 and 3 times higher than the recommended starting doses; "very high doses" were defined as higher than high doses) starting doses of medications, as determined by geriatric pharmacy/ medicine guidelines and expert consensus, was compared by age groups (65-69, 70-74, 75-79, 80-84, and $\geq 85$ years), gender, and hospital.
\end{abstract}

Results: There were 17896 visits representing 11374 unique patients $>65$ years of age $(55.3 \%$ men, $44.7 \%$ women). A total of 3394 doses of medications of interest including 1678 high doses and 684 very high doses were administered to 1364 different patients. Administration of elevated doses of medications was more common than that of recommended doses. Focusing on opioids and BZDs, the 65-69-year age group was much more likely to receive very high doses (1481 and 412 doses, respectively) than the $\geq 85$-year age groups (relative risk [RR] 5.52, 95\% CI 2.56-11.90), mainly reflecting elevated opioid dosing (RR 8.28, 95\% CI 3.69-18.57). Men were more likely than women to receive very high doses (RR 1.47, 95\% CI 1.26-1.72), primarily due to BZDs (RR 2.12, 95\% CI 2.07-2.16).

Conclusion: Administration of elevated doses of opioids and BZDs in the older population occurs frequently in the ED, especially to the 65-69-year age group and men. Further attention to potentially unsafe dosing of high-risk medications to older adults in the ED is warranted.

Keywords: emergency department, older adults, administration, NSAIDs, benzodiazepines, opioids

\section{Introduction}

The population aged 65 years and older is projected to double between 2010 and 2050 . The older adult population is particularly susceptible to the adverse effects of a variety of medications due to physiologic changes, polypharmacy, and comorbid medical conditions. These factors add complexity to the decision making of administering medications to treat their primary complaint or to facilitate further medical treatment in the emergency department (ED). With the aging of the general public, older adult patients have become a growing population in the ED. Adults older than 65 years (older adults) currently account for $15 \%$ of the general population; yet, they are overrepresented in the ED population, making up to $20 \%$ of all ED visits. This percentage will grow as the population ages. ${ }^{1-3}$ Older patients present to the ED at a higher rate than any other
Correspondence: Mitchell Kim Department of Emergency Medicine, University of Washington, 3259 th Avenue Box 359702, Seattle, WA 98104, USA

Email mitchkim87@gmail.com 
age group except for infants. ${ }^{4}$ Moreover, older adults are admitted to the hospital more frequently than any other age group, due to higher acuity and more chronic comorbidities than their younger counterparts. ${ }^{4}$

Opioids, benzodiazepines (BZDs), and nonsteroidal anti-inflammatory drugs (NSAIDs) can cause significant morbidity in older patients when administered in doses typically given to younger adults. ${ }^{5}$ Opioids have been shown to increase the risk of deleterious outcomes, including falls. ${ }^{6,7}$ BZDs have also been associated with increased falls, hospitalization for falls, and hip fractures in older patients. In older adults, BZDs are implicated in approximately $10 \%$ of all drug-associated admissions. ${ }^{8}$ The use of NSAIDs increases the risk of peptic ulcer disease in a dose-response fashion. NSAIDs also increase the risk of fluid retention, hypertension, and acute kidney injury. ${ }^{9}$ Polypharmacy and drug-drug interactions further complicate the extent of potential harm in this population.

High-risk analgesics and anxiolytics are often clinically indicated in the ED and account for two of the five most frequently prescribed classes of medications. Morphine, hydromorphone, ibuprofen, ketorolac, and lorazepam are among the medications most prescribed in the ED. ${ }^{10}$ There are conflicting data on the prevalence of undertreating older adults' pain, suggesting a tension between the risk of adverse drug events and adequate symptomatic relief. ${ }^{11,12}$ It is difficult to strike a balance between alleviating pain or anxiety and avoiding adverse drug events related to these medications. Moreover, when titrated carefully, it has been shown that higher dose of opioids can be safely administered to older persons in the ED. ${ }^{13}$

The literature on the administration of potentially unsafe doses of high-risk medications for pain and anxiety in the older adult in the ED is limited. Most studies have focused on medications that bring older patients into the ED. ${ }^{3,6}$ Little is known regarding how frequently these medications are actually used and their associated average and range of doses. The patient or hospital characteristics correlated with administration of elevated doses (compared to the recommended geriatric dosing guidelines) have not been well studied. This study investigates key patient characteristics associated with very high dosing of medications for pain and anxiety to the older adult population in the ED.

\section{Patients and methods Study design and setting}

We conducted a cross-sectional study of high-risk medications administered to adults $\geq 65$ years of age in the EDs of two academic medical centers in Seattle, WA, during a 6-month period (August 17, 2014, to February 17, 2015). The University of Washington Human Subjects Division, institutional review board (IRB) \#49128, approved this study.

The medication administration database was queried for classes of medications commonly used for pain, anxiety, and behavioral disturbances in the ED setting. This database included only de-identified information. Exclusions included the following: classes of medications representing less than $5 \%$ of all high-risk medication doses given to older patients (e.g., antipsychotics) and administration via uncommon routes (e.g., transdermal and rectal). Individual medications were categorized by class as opioids, BZDs, or NSAIDs. Recommended and elevated doses were determined based on consensus among: 1) established recommended geriatric dosing from two separate online prescribing sites routinely used by clinicians for dosing assistance ${ }^{14,15}$ and 2) individual review by a pharmacist with specific expertise in geriatric pharmacy and two internal medicine board-certified geriatricians. ${ }^{16,17}$ Elevated doses were further subdivided into "high doses" and "very high doses." High doses were defined as doses 1.5-3 times higher than the recommended dose, and very high doses were defined as higher than high dose. For all but four medications, very high dose was greater than three times the recommended dose (Table 1). Dose ranges were also defined by the actual formulations available for administration in the ED (Table 1). Since the definition of what constitutes a "high dose" was more variable during the establishment of consensus, subsequent data analyses focused on "very high doses" of the medications of interest (opioids, BZDs, and NSAIDs).

\section{Covariates}

For each medication administration, age, gender, race, and hospital of the patient receiving the dose were also collected from the medication administration database. Patient age was categorized by the 5-year age group (65-69, 70-74, 75-79, $80-84$, and 85 years or older). Race was categorized as follows: American Indian, Asian, Black, Hispanic, or White. The hospitals are two academic medical centers in Seattle, WA, and were randomly labeled " $\mathrm{A}$ " or " $\mathrm{B}$ ". Hospital A is a regional transplant, cardiac care, and oncologic treatment center. Hospital B is the regional level 1 trauma center and safety net hospital. Both hospitals have a large primary care population that utilizes their ED for urgent issues, similar access to an ED-based pharmacist, are served by the same attending and resident physician group, and share an identical electronic ordering infrastructure. 
Table I Medications of interest by class and individual name with route and dose categories

\begin{tabular}{|c|c|c|c|}
\hline \multirow[t]{2}{*}{ Medications of interest } & \multirow[t]{2}{*}{ Recommended dose } & \multicolumn{2}{|c|}{ Elevated doses } \\
\hline & & High & Very high \\
\hline \multicolumn{4}{|l|}{ Opioids } \\
\hline Fentanyl (IV), mg & $10-25$ & $37.5-75$ & $>75$ \\
\hline Hydromorphone (IV/IM), mg* & $0.05-0.4$ & $0.5-0.9$ & $>0.9$ \\
\hline Hydromorphone (PO), mg & 1 & $1.5-3$ & $>3$ \\
\hline Morphine (IV/IM), mg* & $0.5-2$ & $3-5$ & $>5$ \\
\hline Morphine (PO), mg & $10-15$ & $30-45$ & $>45$ \\
\hline Oxycodone (PO), mg & $2.5-5$ & $7.5-15$ & $>15$ \\
\hline \multicolumn{4}{|l|}{ BZDs } \\
\hline Chlordiazepoxide (PO), mg & 10 & $15-30$ & $>30$ \\
\hline Clonazepam (PO), mg & 0.25 & $0.375-0.75$ & $>0.75$ \\
\hline Diazepam (IV/IM, PO), mg & 2.5 & $3.75-7.5$ & $>7.5$ \\
\hline Lorazepam (IV/IM, PO), mg & $0.25-0.5$ & $0.75-1.5$ & $>1.5$ \\
\hline Midazolam (IV), mg & $0.25-0.5$ & $0.75-1.5$ & $>1.5$ \\
\hline \multicolumn{4}{|l|}{ NSAIDs } \\
\hline Ibuprofen (PO), mg* & 400 & $600-800$ & $>800$ \\
\hline Ketorolac (IV/IM), mg & $10-15$ & $30-45$ & $>45$ \\
\hline Naproxen (PO), mg* & 375 & $500-750$ & $>750$ \\
\hline
\end{tabular}

Note: *High dose range is smaller than 1.5-3.0 times the maximum recommended dose.

Abbreviations: BZDs, benzodiazepines; NSAIDs, nonsteroidal anti-inflammatory drugs; IV, intravenous; IM, intramuscular; PO, by mouth.

\section{Outcomes}

In this cross-sectional study, the primary outcome measure was relative risk (RR) of receiving a very high medication dose.

\section{Statistical analysis}

We calculated descriptive statistics detailing the medication administrations of interest, stratifying medication class by each of the covariates: age group, gender, and hospital. Univariate comparisons were made using Pearson's chisquared test or Fisher's exact test, as appropriate. Unadjusted RRs of very high medication dosing were calculated for all medications and individually for each medication class. Logbinomial regression models were constructed with hospital clustering to estimate adjusted RRs of very high dosing for all medications and individually for each medication class. ${ }^{18}$ Model performances were evaluated with the Wald test for specification. Significance for statistical hypotheses was set at alpha $=0.05$. All calculations were performed using Stata/ SE 12.1 (StataCorp LP, College Station, TX, USA); graphics were generated with Prism 6.01 (GraphPad Software, Inc., La Jolla, CA, USA).

\section{Results}

A total of 17896 visits, representing 11374 unique patients aged 65 years and older, were identified at both centers during the study period. Of the unique patients, $55.3 \%$ were men and $44.7 \%$ were women. In addition, $63.1 \%$ of all unique patients presented to hospital $\mathrm{A}$ and $36.9 \%$ of all unique patients presented to hospital B (Figure 1).

A total of 3394 doses of medications of interest (opioids, BZDs, NSAIDs) were administered to 1364 unique patients. A total of 2362 doses were elevated doses, 1678 were high doses, and 684 were very high doses. There were 88 encounters during the study period involving intubated patients in the ED (intubated pre-hospital or in the ED). An additional 14 encounters involved patients undergoing procedural sedation. The majority of patients who were intubated in our study period were patients at hospital B (79 patients at $\mathrm{B}$, nine patients at $\mathrm{A}$ ). The numbers of procedural sedations were nearly equal at each center (six patients at $\mathrm{B}$, eight patients at A). These patients made up a small minority of all patients, but remained in the analysis and may have received elevated doses more frequently than others given their medical condition. Table 2 summarizes the frequency of total doses of medications of interest that were given, separated by age group, race, gender, hospital, and drug class. No racial group, except non-Hispanic and non-Latino White, represented more than $10 \%$ of the study population (i.e., administered doses). Further stratification by race was not performed. The medications of interest were prescribed to women and men with near equal frequency ( $47.2 \%$ versus $52.8 \%$ ). Opiates were prescribed most frequently $(80.2 \%)$, followed by BZDs $(14.3 \%)$ and NSAIDs (5.5\%). Notably, each medication group was given more often at elevated doses than at recommended doses (Table 3). Despite having fewer visits from patients 


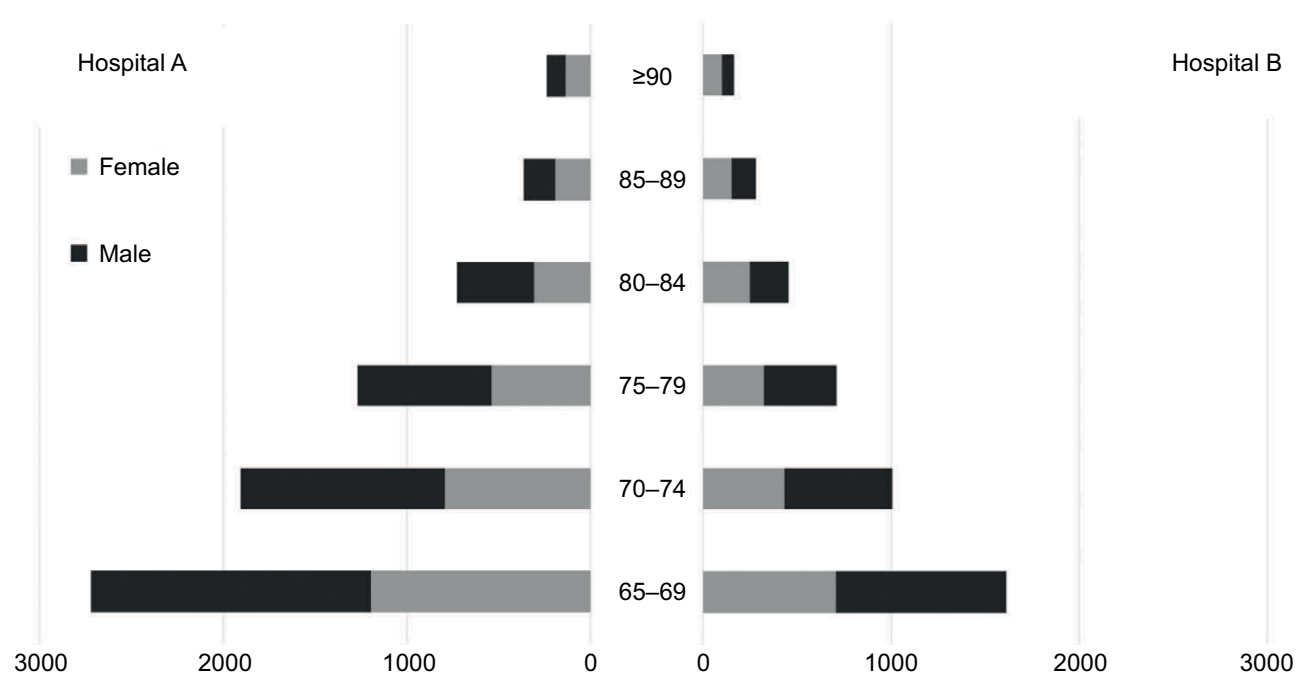

Figure I The number of unique individuals, by gender and age group, who presented to each of the study center EDs. Abbreviation: ED, emergency department.

aged 65 years and older, hospital B had over twice the number of doses of medications of interest given as hospital A. The median number of visits made by an individual patient during the study period was 2 (interquartile range of $1-3$; 95th percentile 6). These patients received a median of two doses per visit (interquartile range of $1-3 ; 95$ th percentile 6 ). Of all medications, fentanyl, hydromorphone, and midazolam were most frequently re-dosed (median of two doses; interquartile range 1-3; data not shown).

We then focused on very high doses of the medications of interest. Of 3394 doses provided for 1364 unique patients, 684 doses were considered to be very high. There was a significantly increased risk of receiving a very high dose of opioid and BZD medications in the 65-69-year age group compared to those aged 85 years and older (RR for all medications: $5.52,95 \%$ CI 2.56-11.90; opioids: $8.28,95 \%$ CI 3.69-18.57; BZDs: $1.74,95 \%$ CI 1.57-1.92; there were insufficient data on very high doses of NSAIDs). Opioids were the class of medications that had the strongest trend toward very high dosing in the younger age groups, with an RR for receiving a very high dose inversely correlated with age (Table 4).

In addition, men were more likely than women to receive a very high dose of all study drugs combined (RR 1.47, 95\% CI 1.26-1.72), opioids (RR 1.29, 95\% CI 1.22-1.36), and BZDs (RR 2.12, 95\% CI 2.07-2.16). Again, there were insufficient numbers of very high doses of NSAID to specifically comment on gender differences in this drug category. There was a trend toward increased risk of very high doses of all medications of interest at hospital B compared to hospital A that was not statistically significant. This reflected less use
Table 2 Total doses (\% of all doses) of medications of interest administered by age group, race, gender, hospital, and drug class

\begin{tabular}{ll}
\hline Characteristic & $\mathbf{n}(\%)$ \\
\hline Age group (years) & $148 \mid(43.6)$ \\
$65-69$ & $717(2 I .1)$ \\
$70-74$ & $470(13.9)$ \\
$75-79$ & $343(10.1)$ \\
$80-84$ & $383(11.3)$ \\
$85+$ & \\
Race/ethnicity & $87(2.6)$ \\
American Indian or Alaskan native & $316(9.3)$ \\
Asian & $305(9.0)$ \\
Black & $15(0.4)$ \\
Native Hawaiian or other Pacific Islander & $77(2.3)$ \\
Hispanic or Latino White & $2594(76.4)$ \\
Non-Hispanic, non-Latino White & \\
Gender & $1603(47.2)$ \\
Female & $179 \mid(52.8)$ \\
Male & \\
Hospital & $1062(3 I .3)$ \\
A & $2332(68.7)$ \\
B & $2722(80.2)$ \\
Drug class & $484(14.3)$ \\
Opioid & $188(5.5)$ \\
BZDs &
\end{tabular}

Abbreviations: BZDs, benzodiazepines; NSAIDs, nonsteroidal anti-inflammatory drugs.

of very high doses of opioids and greater use of very high doses of BZDs administered at hospital B (Table 4, Figure 2).

\section{Discussion}

We evaluated the frequency with which high-risk groups of medications are utilized and the characteristics associated with elevated dosing, with a focus on very high doses, of these 


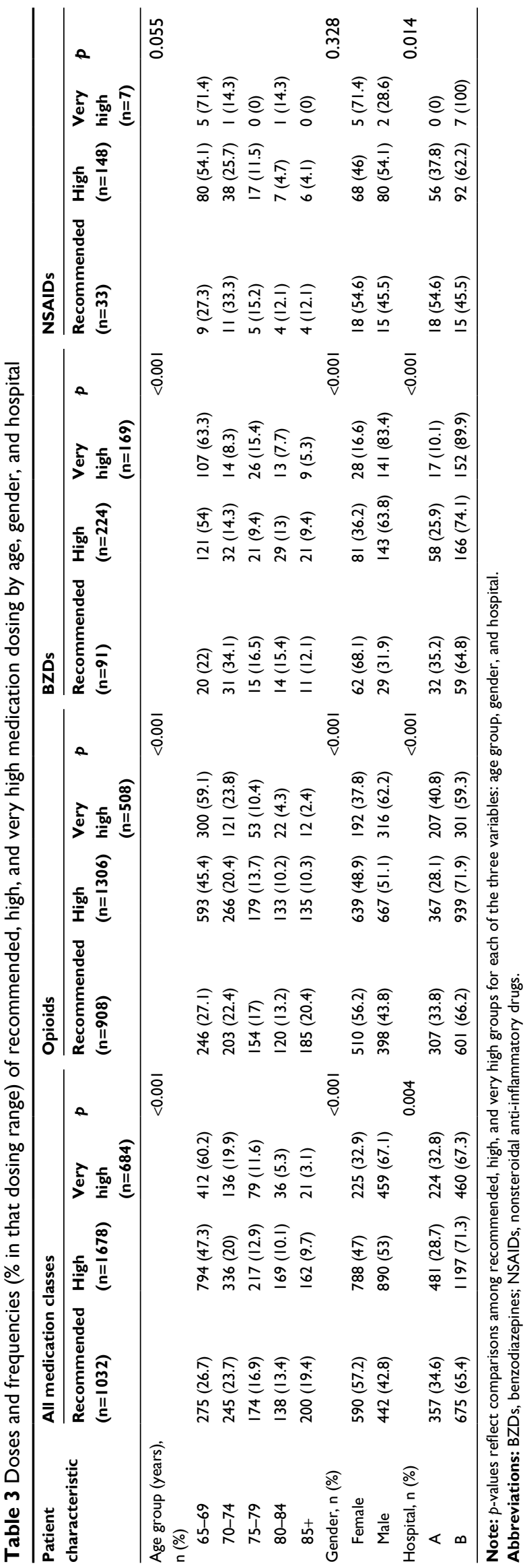

medications in the older adult population at two academic EDs. We found that very high doses of high-risk medications were frequently administered, especially to patients in the younger age group and men.

Medication management is a top priority in the care of older adults presenting to the ED. ${ }^{19}$ Most research has focused on medications taken as outpatients that then led to ED visits, use of medications for specific ED situations, and medication management at ED discharge. ${ }^{6,12,20,21}$ Few studies have evaluated potentially inappropriate doses of medications administered while in the ED. Nixdorff et $\mathrm{al}^{22}$ noted that $6.5 \%$ of older patients in the ED received an "inappropriate medication" (defined by 2003 Beers Criteria), whereas 3.6\% of patients received an "inappropriate medication" (defined by 1997 Beers Criteria) in Chin et al's ${ }^{23}$ prospective cohort of 898 older patients. Tools such as the screening tool for older people's prescriptions (STOPP) Criteria, which screens for use of medications including opioids, BZDs, and NSAIDs in older adults, have not been studied in the ED population. ${ }^{24}$ Our results showed that very high doses of opiates were prescribed most frequently, followed by BZDs and NSAIDs. The use of opioids likely reflects the need for these medications for acute pain control in the ED setting. Consequently, it is not surprising that in absolute numbers opioids were also more likely to be given at elevated doses than either BZDs or NSAIDs.

The risk of receiving a very high dose of a medication of interest diminished with increasing age among the older populations. Accordingly, this risk was highest in the 65-69-year age group and generally decreased in the older age groups. This change with advancing age was most notable in the risk of receiving very high doses of opioids (RR 8.28) and was less pronounced with BZDs (RR 1.74). These data are consistent with previous research that has shown a decrease in ED use of analgesic medications, and opioids in particular, with advancing age. ${ }^{12,25,26}$ These previous studies did not specifically evaluate the administration of these medications in older age groups while in the ED. Nonetheless, prior work and the data presented in this study suggest that there is recognition of the vulnerability of the $\geq 85$-year age group to medications, even in emergency settings where higher doses of analgesics and anxiolytics are often warranted.

There is a growing body of literature regarding gender disparities and the frequency of dosing of analgesics in the ED. However, the gender differences on the amount of medication given with each administration have not been studied. We found a difference based upon gender wherein men received 
Table 4 Unadjusted and adjusted RRs of very high dosing versus recommended dosing of medications of interest

\begin{tabular}{|c|c|c|c|c|c|c|}
\hline \multirow{2}{*}{$\begin{array}{l}\text { Patient } \\
\text { characteristic }\end{array}$} & \multicolumn{2}{|c|}{ All medication classes ${ }^{a}$} & \multicolumn{2}{|l|}{ Opioids } & \multicolumn{2}{|l|}{ BZDs } \\
\hline & Unadjusted & Adjusted & Unadjusted & Adjusted & Unadjusted & Adjusted \\
\hline \multicolumn{7}{|l|}{ Age group (years) } \\
\hline $65-69$ & $6.31(4.79-8.32)^{* *}$ & $5.52(2.56-11.90)^{* *}$ & $9.02(6.28-12.96)^{* *}$ & $8.28(3.69-18.57)^{* *}$ & $1.87(1.38-2.55)^{* *}$ & $1.74(1.57-1.92)^{* *}$ \\
\hline $70-74$ & $3.76(2.60-5.43)^{* *}$ & $3.57(1.03-12.33)^{*}$ & $6.13(3.92-9.60)^{* *}$ & $5.87(1.70-20.27)^{*}$ & $-0.14(-0.39-0.11)$ & $0.94(0.87-1.01)$ \\
\hline $75-79$ & $3.29(2.19-4.92)^{* *}$ & $3.11(1.40-6.93)^{*}$ & $4.20(2.48-7.13)^{* *}$ & $4.12(1.67-10.19)^{*}$ & $\mathrm{I} .4 \mathrm{I}(0.86-2.32)$ & $1.58(|.47-| .7 \mid)^{* *}$ \\
\hline $80-84$ & $2.18(1.34-3.54)^{*}$ & $2.14(0.84-5.46)$ & $2.54(1.34-4.85)^{*}$ & $2.55(0.54-12.02)$ & $1.07(0.57-2.00)$ & $1.47(1.12-1.94)^{*}$ \\
\hline $85+$ & Reference & Reference & Reference & Reference & Reference & Reference \\
\hline \multicolumn{7}{|l|}{ Gender } \\
\hline Female & Reference & Reference & Reference & Reference & Reference & Reference \\
\hline Male & $1.85(1.63-2.08)^{* *}$ & $1.47(1.26-1.72)^{* *}$ & $1.62(1.40-1.87)^{* *}$ & $1.29(1.22-1.36)^{* *}$ & $2.67(2.12-3.36)^{* *}$ & $2.12(2.07-2.16)^{* *}$ \\
\hline \multicolumn{7}{|l|}{ Hospital } \\
\hline$A$ & Reference & Reference & Reference & Reference & Reference & Reference \\
\hline B & $1.05(0.93-1.19)$ & $1.02(0.98-1.07)$ & $0.83(0.72-0.95)^{*}$ & $0.85(0.84-0.87)^{* *}$ & $2.08(1.55-2.78)^{* *}$ & I.8I (I.78-I.83)** \\
\hline
\end{tabular}

Note: Data are presented as RR $(95 \% \mathrm{Cl}) ;{ }^{*} p<0.00 \mathrm{I} ;{ }^{*} p<0.05$. $p$-values reflect comparisons between the reference group and each of the other within-variable groups. aNSAIDs had insufficient numbers for risk calculation as a separate group.

Abbreviations: BZDs, benzodiazepines; NSAIDs, nonsteroidal anti-inflammatory drugs; RR, relative risk.

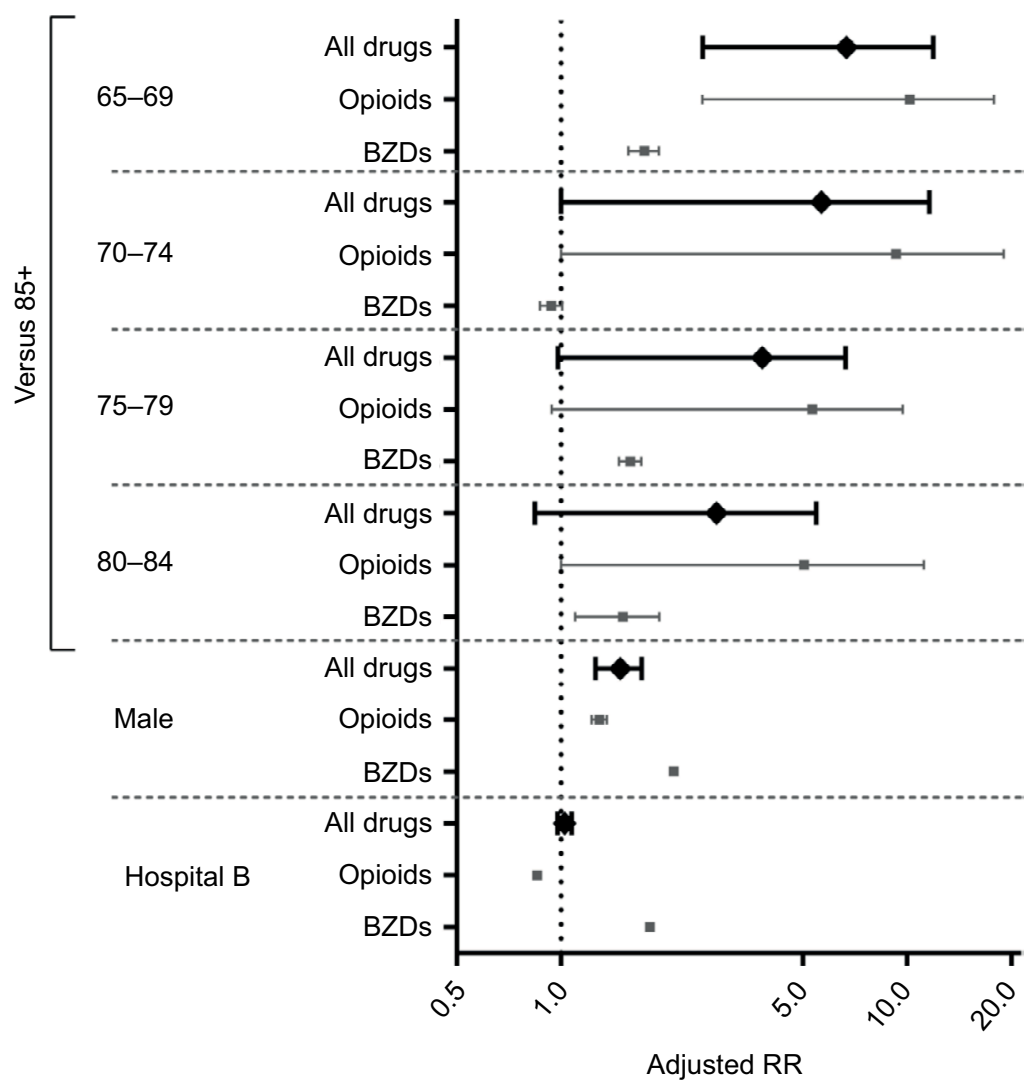

Figure 2 Forest plot of the adjusted RRs of receiving a very high dose for all medication groups.

Note: NSAIDs had insufficient numbers for risk calculation as a separate group.

Abbreviations: BZDs, benzodiazepines; NSAIDs, nonsteroidal anti-inflammatory drugs; RR, relative risk.

very high doses of medications of interest more frequently than women. This was most pronounced with BZDs. On the other hand, women were more likely than men to receive any dose of analgesic while in the ED as summarized in Table 3. Although women made up $47.2 \%$ of all patients, they received $49.2 \%$ of all opioid doses and $48.4 \%$ of all NSAID doses.
These data are consistent with several prospective studies in the ED that have suggested that adult women of all ages are more likely to report pain, are perceived to be in more pain, and receive more doses of any analgesics (including opioids and NSAIDs) than men. ${ }^{27-29}$ Our results corroborate that older women received more frequent doses of analgesics, but the 
doses of medications administered were generally lower than that given to men.

To our knowledge, there have been no studies evaluating the frequency or dosing of BZD administration in the older adult population in the ED. Previous research has focused on outpatient BZD prescriptions, which appear to be more frequently given to women than men from both clinics and EDs. ${ }^{20}$ This follows the trend that anxiety disorders are twice as prevalent in women than in men. In a study by Gleason et al, ${ }^{30}$ the odds ratio of BZD use in community-dwelling older adult population was 1.72 for women. This is in contrast to our study, which shows that women are less frequently administered BZDs within the $\mathrm{ED}$ and received lower doses than men (RR for very high dose 2.12). This difference may reflect that older men more frequently require acute administration of BZDs at higher doses for indications such as alcohol withdrawal, which is more common in men. ${ }^{31}$

The overall rate (in number of doses per unique patient visit) of administration of medications of interest was over two times higher at hospital B than hospital A. Hospital B was more likely to administer very high doses of BZDs, but not opioids, than hospital A. Hospital B serves as a regional level 1 trauma and burn center while also serving as a county safety-net hospital. In contrast, hospital A serves as a regional quaternary care referral hospital. It is likely the differences in BZD administration largely reflect the patient population, such as the trauma patients at hospital B, which results in a far greater number of intubations. As previously noted, each hospital has a large primary care population and is served by the same resident and attending physician group, each shares an identical electronic ordering infrastructure, and each has an ED-based pharmacist for 10 hours per day. The literature is sparse on the role of hospital characteristics on ED administration of these medications. There does not appear to be an association between ED BZD prescription trends and the hospital region, profit status, locale (metropolitan versus rural), or teaching versus nonteaching status. ${ }^{20}$ However, our findings indicate that the hospital setting, and the patients that they serve, influences the pattern of actual administration of certain medications in the ED.

There are several limitations to our retrospective database analysis. First, the generalizability of our findings to nonacademic practice settings is unclear. While we did account for medication administration related to procedural sedations and intubations, other case-by-case ordering indications were not analyzed. As our goal was to define overall patterns of administration of high-risk medications, we did not specifically investigate whether there was incremental dose escalation versus initial elevated dosing, or subsequent adverse events. Concurrent medication usage and medical comorbidities, such as the use of chronic pain medications that might inform dosing choices, was not evaluated. Nonetheless, this study provides the foundation for creating tools to provide guidance on the dosage and administration of potentially high-risk medications to the older population in the ED.

\section{Conclusion}

We found opioids and BZDs are often administered at very high doses to those 65 years and older in the ED. The risk for receiving a very high dose decreases with increasing age and is greatest in those aged 65-69 years. Women receive more doses of analgesic medications, but men are more likely to receive very high doses of opioids and BZDs. In addition, the specialization of a hospital facility and the characteristics of the patient population are factors that influence the frequency and doses of the medications administered in the ED. We acknowledge that the ED setting often warrants higher doses of analgesics and anxiolytics, but our data describe a common practice of administering high-risk medications at very high doses to older adults. Given these findings, interventions aimed at age-specific dosing guidelines for the $\mathrm{ED}$ merit further examination.

\section{Key points}

1. Administration of elevated doses of high-risk medications for pain and anxiety in older adults occurs frequently in the emergency department.

2. The risk of receiving a very high dose of an opioid or a benzodiazepine decreases with increasing age.

3. Patients in the 65-69-year age group and men were at greatest risk for receiving very high doses of opioids and benzodiazepines.

\section{Acknowledgments}

The authors thank Dr Susan A Stern, Department of Emergency Medicine, University of Washington, for her support of this project and Robert Anthony Black and Christine T Fong of the National Center for Advancing Translational Sciences of the National Institutes of Health under award number UL1 TR000423 for assistance with data collection. This work was supported by the John A Hartford Foundation (to MJR).

\section{Disclosure}

The authors report no conflicts of interest in this work. 


\section{References}

1. Federal Interagency Forum on Aging-Related Statistics. Older Americans 2016, Key indicators of well-being. Washington, DC: Federal Interagency Forum on Aging-Related Statistics; 2016. Available from: https://aging stats .gov/docs/LatestReport/Older-Americans-2016-KeyIndicators-of-WellBeing.pdf. Accessed August 12, 2017.

2. Hwang U, Shah MN, Han JH, Carpenter CR, Siu AL, Adams JG. Transforming emergency care for older adults. Health Aff. 2013;32(12): 2116-2121.

3. Samaras N, Chevalley T, Samaras D, Gold G. Older patients in the emergency department: a review. Ann Emerg Med. 2010;56(3):261-269.

4. Weiss AJ, Wier LM, Stocks C, Blanchard J. Overview of Emergency Department Visits in the United States, 2011: Statistical Brief \#174. Rockville, MD: Healthcare Cost and Utilization Project (HCUP) Statistical Briefs; 2006.

5. Alassaad A, Melhus H, Hammarlund-Udenaes M, Bertilsson M, Gillespie U, Sundstrom J. A tool for prediction of risk of rehospitalisation and mortality in the hospitalised elderly: secondary analysis of clinical trial data. BMJ Open. 2015;5(2):e007259.

6. Buckeridge D, Huang A, Hanley J, et al. Risk of injury associated with opioid use in older adults. J Am Geriatr Soc. 2010;58(9):1664-1670.

7. Terrell KM, Heard K, Miller DK. Prescribing to older ED patients. Am J Emerg Med. 2006;24(4):468-478.

8. Madhusoodanan S, Bogunovic OJ. Safety of benzodiazepines in the geriatric population. Expert Opin Drug Saf. 2004;3(5):485-493.

9. McCarberg BH. NSAIDs in the older patient: balancing benefits and harms. Pain Med. 2013;14(suppl 1):S43-S44.

10. Centers for Disease Control and Prevention [webpage on the Internet]. National Hospital Ambulatory Medical Care Survey: 2010. Atlanta, GA: Emergency Department Summary Tables. 2013. Available from: http:// www.cdc.gov/nchs/ahcd.htm. Accessed January 3, 2016.

11. Cinar O, Ernst R, Fosnocht D, et al. Geriatric patients may not experience increased risk of oligoanalgesia in the emergency department. Ann Emerg Med. 2012;60(2):207-211.

12. Platts-Mills TF, Esserman DA, Brown DL, Bortsov AV, Sloane PD, McLean SA. Older US emergency department patients are less likely to receive pain medication than younger patients: results from a national survey. Ann Emerg Med. 2012;60(2):199-206.

13. Chang AK, Bijur PE, Davitt M, Gallagher EJ. Randomized clinical trial of an intravenous hydromorphone titration protocol versus usual care for management of acute pain in older emergency department patients. Drugs Aging. 2013;30(9):747-754.

14. Truven Health Analytics LLC [webpage on the Internet]. Micromedex Solution: Web Application Access; 2012-2016. Available from: https:// www.micromedexsolutions.com/home/dispatch/ssl/true. Accessed January 3, 2016.

15. UpToDate [database on the Internet]. 2016. Available from: http://www. uptodate.com/contents/search. Accessed January 3, 2016.

16. Gardner DM, Murphy AL, O'Donnell H, Centorrino F, Baldessarini RJ. International consensus study of antipsychotic dosing. Am J Psychiatry. 2010;167(6):686-693.
17. Mattison ML, Catic A, Davis RB, et al. A standardized, bundled approach to providing geriatric-focused acute care. J Am Geriatr Soc. 2014;62(5):936-942.

18. Barros AJ, Hirakata VN. Alternatives for logistic regression in crosssectional studies: an empirical comparison of models that directly estimate the prevalence ratio. BMC Med Res Methodol. 2003;3:21.

19. Carpenter CR, Bromley M, Caterino JM, et al. Optimal older adult emergency care: introducing multidisciplinary geriatric emergency department guidelines from the American College of Emergency Physicians, American Geriatrics Society, Emergency Nurses Association, and Society for Academic Emergency Medicine. Acad Emerg Med. 2014;21(7):806-809.

20. Marra EM, Mazer-Amirshahi M, Brooks G, van den Anker J, May L, Pines JM. Benzodiazepine prescribing in older adults in U.S. Ambulatory Clinics and Emergency Departments (2001-10). J Am Geriatr Soc. 2015;63(10):2074-2081.

21. Stevens MB, Hastings SN, Powers J, et al. Enhancing the quality of prescribing practices for older veterans discharged from the Emergency Department (EQUiPPED): preliminary results from Enhancing Quality of Prescribing Practices for older veterans discharged from the Emergency Department, a novel multicomponent interdisciplinary quality improvement initiative. J Am Geriatr Soc. 2015;63(5):1025-1029.

22. Nixdorff N, Hustey FM, Brady AK, Vaji K, Leonard M, MessingerRapport BJ. Potentially inappropriate medications and adverse drug effects in elders in the ED. Am J Emerg Med. 2008;26(6):697-700.

23. Chin MH, Wang LC, Jin L, et al. Appropriateness of medication selection for older persons in an urban academic emergency department. Acad Emerg Med. 1999;6(12):1232-1242.

24. Hamilton H, Gallagher P, Ryan C, Byrne S, O'Mahony D. Potentially inappropriate medications defined by STOPP criteria and the risk of adverse drug events in older hospitalized patients. Arch Intern Med. 2011;171(11):1013-1019.

25. Jones JS, Johnson K, McNinch M. Age as a risk factor for inadequate emergency department analgesia. Am J Emerg Med. 1996;14(2): 157-160.

26. Mills AM, Edwards JM, Shofer FS, Holena DN, Abbuhl SB. Analgesia for older adults with abdominal or back pain in emergency department. West J Emerg Med. 2011;12(1):43-50.

27. Chen EH, Shofer FS, Dean AJ, et al. Gender disparity in analgesic treatment of emergency department patients with acute abdominal pain. Acad Emerg Med. 2008;15(5):414-418.

28. Raftery KA, Smith-Coggins R, Chen AH. Gender-associated differences in emergency department pain management. Ann Emerg Med. 1995;26(4):414-421.

29. Safdar B, Heins A, Homel P, et al. Impact of physician and patient gender on pain management in the emergency department - a multicenter study. Pain Med. 2009;10(2):364-372.

30. Gleason PP, Schulz R, Smith NL, et al. Correlates and prevalence of benzodiazepine use in the community-dwelling elderly. $J$ Gen Intern Med. 1998;13:243-250.

31. Erol A, Karpyak VM. Sex and gender-related differences in alcohol use and its consequences: contemporary knowledge and future research considerations. Drug Alcohol Depend. 2015;156:1-13.
Drug, Healthcare and Patient Safety

\section{Publish your work in this journal}

Drug, Healthcare and Patient Safety is an international, peer-reviewed open access journal exploring patient safety issues in the healthcare continuum from diagnostic and screening interventions through to treatment, drug therapy and surgery. The journal is characterized by the rapid reporting of reviews, original research, clinical, epidemiological and post-marketing surveillance studies, risk management, health literacy and educational programs across all areas of healthcare delivery. The manuscript management system is completely online and includes a very quick and fair peer-review system. Visit http://www.dovepress.com/ testimonials.php to read real quotes from published authors. 